\title{
Passive position error correction in internet-based teleoperation *
}

\author{
Alejandro Fernández Villaverde ${ }^{\mathrm{a}}$, Antonio Barreiro ${ }^{\mathrm{b}}$, Cesáreo Raimúndez ${ }^{\mathrm{b}}$ \\ ${ }^{a}$ Bioprocess Engineering Group, IIM (CSIC). Eduardo Cabello, 6, 36208 Vigo, Spain \\ ${ }^{\mathrm{b}}$ Department of Systems \& Control, ETSEI (University of Vigo). Campus Universitario, 36310 Vigo, Spain
}

\begin{abstract}
During the last two decades, important advances have been made in the field of bilateral teleoperation. Different techniques for performing stable teleoperation in non-ideal conditions have been developed, especially in a passivity framework. Until recently, however, no robust solutions for addressing this problem with variable delays and other drawbacks of packet-switched networks have been developed. The requirement of maintaining passivity in these circumstances degrades performance, due to the loss of energy that it involves. In this paper an arrangement is proposed which is capable of eliminating position errors, while maintaining passivity of an internet-like channel. The behaviour of this new controller is studied by Lyapunov analysis, compared to previous methods, and validated through numerical simulations.
\end{abstract}

Key words: Teleoperation; Passive compensation; Control algorithms; Discrete time; Telerobotics; Time delay.

\section{Introduction}

Teleoperated systems follow a master-slave scheme, in which a slave manipulator reproduces the movements of a master manipulator commanded by an operator. In bilateral teleoperation [7] force feedback is provided to the operator, enabling him to feel the contacts with the environment. Modern bilateral teleoperation stems from [1], where it was shown that if master and slave exchange power variables (such as force and velocity), the communications channel is not passive in presence of time delays and may destabilize the whole system. The proposed solution was reformulated in [9] as the transmission of a pair of wave variables, which preserves passivity of the communications channel for constant time delays. Position error may arise due to different reasons (initial mismatch, contacts with the environment, numerical errors...); and, since only the master velocity is transmitted-not its position-there is no way to recover from this error. Several improvements have been pro-

\footnotetext{
^ This paper was partially presented at the 17th IFAC World Congress, July 6-11 2008, Seoul, South Korea. Corresponding author A.F. Villaverde, Ph. +34 986 231930, Fax +34 986292762.

Email addresses: afvillaverde@iim.csic.es (Alejandro Fernández Villaverde), abarreiro@uvigo.es (Antonio Barreiro), cesareo@uvigo.es (Cesáreo Raimúndez).
}

posed to overcome this problem. In [11] it was suggested to transmit the integrals of the wave variables, which encode position and momentum information. A modification of the wave commands [10] was also proposed. In [16] a similar method was presented, introducing the idea of using the energy produced by this solution to reduce the position error that appears after a communication blackout. In [4] a modified architecture was proposed in which additional position signals were transmitted and proportional controllers were added on each side. A condition for stability of these controllers for constant time delays was given. In [14] a method for compensating position errors in port-hamiltonian bilateral teleoperation with constant time delays was proposed. The slave controller is modified by adding a virtual tank, which stores the energy dissipated in the resistive element. The state of the controller is augmented with a variable corresponding to the rest length of its elastic element, which is changed in order to compensate for the position error, while the required energy is extracted from the tank. In a recent extension [15] this arrangement was modified to compensate for the errors caused by packet losses in the network, as well as by dissipation in the impedance controller. In [2] different wave transformation arrangements, resulting in admittancetype and hybrid-type teleoperation architectures, were examined. Some approaches that are not based on scattering have also appeared: in [12] a scheme encoding po- 
sition and integral of force was proposed, and in $[5,13]$ the passivity-based architecture was extended to guarantee state synchronization of master/slave robots. Some common assumptions of these methods, such as constant time delays, or that the data arrives in the same order it is transmitted, limit their application.

The contribution of this paper is the development of a controller for improving position tracking in bilateral teleoperation via packet-switched networks. It is suited for the worst case scenario, i.e. for arbitrary time-varying delays, disordered packets and data losses; and, since it is based on passivity, it doesn't induce instability. The structure of this paper is as follows: first, preliminary considerations are made in section 2 . Then, the controller is presented in section 3 . The overall stability of the setup and the controller's ability to reduce the position error are analyzed in section 4 . Simulation results are shown in section 5 along with some comments, and conclusions are presented in section 6 .

\section{Preliminaries}

Wave variables are the input and output terms $(u, v)$ into which the power flow through a network element's port can be separated:

$u=\frac{1}{\sqrt{2 b}}(F+b \dot{x}), \quad v=\frac{1}{\sqrt{2 b}}(F-b \dot{x})$

Here, $F$ and $\dot{x}$ are power variables (force and velocity), and $b$ represents an impedance. Notice that $\left(u^{2}, v^{2}\right)$ have units of power and, if $h$ is the fixed sampling interval, $u^{2} h, v^{2} h$ represent energy packets.

Suppose we want to use a packet-switched network as communications channel. In [8] it was shown that the UDP protocol is more suitable for control purposes than TCP/IP; however, it fails to guarantee the order of arrival of the data packets, which can also be lost on the way. These problems-variable time delay, disordered packets and data losses-can destabilize the system if not properly treated. In order to preserve passivity, a lost packet should be replaced by a null packet, so that no additional energy is injected into the system. Even if there is no loss of packets, an empty sampling instance can appear due to increasing delay; in this case the same strategy should be adopted. The drawback of this "null packet" strategy is that eliminates more energy than the "previous packet" one, thus degrading performance. In order to improve performance while maintaining passivity, the solution presented in [3] will be adopted. It consists of five modules: Subtractor, Interpolator, Buffer, Compressor and Expander, which are collectively labeled as SIBCE in Fig. 1. When a packet is lost, the interpolator produces a "null packet" instance, but the data contained in the lost packet will be recovered as soon as the next one arrives. When that happens, the energy will be distributed among the number of new samples, producing $n$ samples of value $\hat{u}=\frac{\sum u-\left(\sum u\right)_{\text {previous }}}{t-t_{\text {previous }}}=\frac{\sum_{i=1}^{n} u_{i}}{n}$. The interpolator creates new samples guaranteeing that their energy $\left(E^{\prime}\right)$ is not higher than that of the original samples $(E)$, thus preserving passivity of the communications channel for arbitrary delays and losses:

$E^{\prime}=n \hat{u}^{2} h=\frac{\left(\sum_{i=1}^{n} u(i)\right)^{2} h}{n} \leq E=\sum_{i=1}^{n} u^{2}(i) h$

Assumption 1 From these results, detailed in [3], it can be assumed that for the scheme depicted in Fig. 1, and for the delay buffer in the channel from $u_{m}$ to $v_{s 1}$, the following holds:

$E(k)-E(1) \leq\left[\sum_{i=1}^{k} u_{m}^{2}(i)-\sum_{i=1}^{k} v_{s 1}^{2}(i)\right] h$

where $E(k)$ is the energy stored at time $t=k h$ inside this delay buffer. A similar relation holds for the delay buffer in the channel from $u_{s}$ to $v_{m}$.

\section{Passive position error correction}

\subsection{Exploiting the energy margin}

The previous section's arrangement is energy-dissipating: in order to assure passivity, the energy coming out of the interpolator is equal or less than the incoming energy. Thus, a measurable Energy Margin (EM) is being generated. The correction proposed here consists of reusing this energy margin, injecting it back to the system. This recovered energy is transformed into a correction of the wave variable that is proportional to the position error. The energy of the resulting term is monitored so that it is not increased in a quantity larger than the available EM. In this way, passivity of the communications channel is guaranteed. A diagram of the teleoperation setup is depicted in Fig. 1, where this control action is labelled as "EM" and applies a correction $v_{s 4}$ on the wave variable $v_{s 3}$ (which is obtained after a possible correction $v_{s 2}$ is introduced on $v_{s 1}$ by a "Wave Trimmer" module, WT, to be described later). It is assumed, with no loss of generality, that the master device generates the position setpoint that must be followed by the slave device. The correcting algorithm is as follows:

- At each sampling instance a packet is created at the master side, containing the following data, $\left[\sum_{i=1}^{k} u_{m}(i), E_{m s}(k), x_{m}(k), k\right]$ :

- The sum of all the wave variables generated at the master side until that moment, $\sum_{i=1}^{k} u_{m}(i)$.

- The energy of all the previously sent wave variables, $E_{m s}(k)=\sum_{i=1}^{k} u_{m}^{2}(i) h$ 


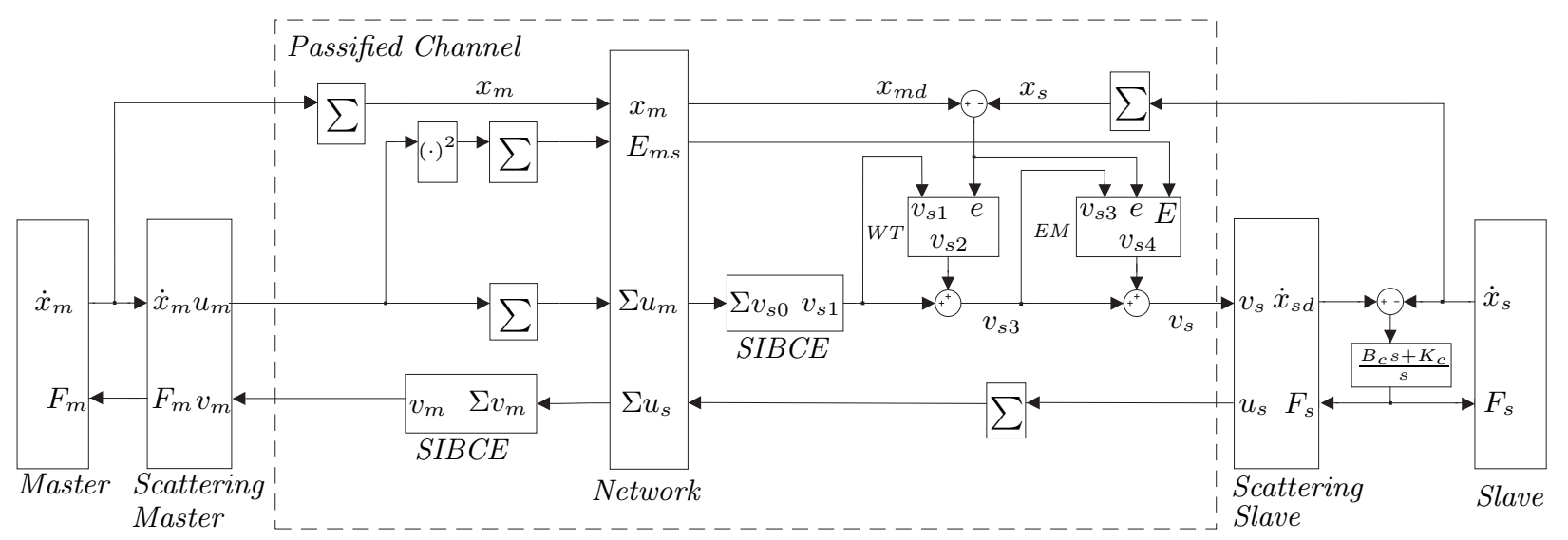

Fig. 1. Overall setup

- The value of the position command, $x_{m}(k)$.

- The sampling instance, $k$.

- This packet is sent through the communications channel to the slave side, where it is read. The position error is then calculated as:

$e(k)=x_{m d}(k)-x_{s}(k)$

where subscript $d$ denotes a delayed signal, i.e. $x_{m d}(k)=x_{m}\left(k-n_{d}\right)$, where $n_{d}$ is the number of sampling instances that the packet is delayed $\left(n_{d}\right.$ is not constant, but we choose to write $n_{d}$ instead of $n_{d}(k)$ for ease of notation). Then, a control term proportional to this position error is obtained as $v_{s}^{\prime}(k)=K_{E M} e(k)$, where the constant $K_{E M}$ is a design parameter. This term is the provisional wave correction and will be added to the value of the received wave variable, $v_{s 3}$, provided that there is some energy margin available. The provisionally modified wave is $v_{s}^{\prime \prime}(k)=v_{s 3}(k)+v_{s}^{\prime}(k)$.

- In order to assure passivity of the communications channel, its outgoing energy must be equal or less than the incoming energy. The energy injected by the modified wave variable is $\left(v_{s}^{\prime \prime}(k)\right)^{2} h$, this is:

$$
E^{\prime}(k)=\left[v_{s 3}(k)+K_{E M}\left(x_{m d}(k)-x_{s}(k)\right)\right]^{2} h
$$

While the energy margin is defined as:

$$
E M(k)=\left[E_{m s}\left(k-1-n_{d}\right)-\sum_{i=1}^{k-1} v_{s 3}^{2}(i)\right] h
$$

If the injected energy does not make the energy balance from $v_{s 3}$ to $v_{s}$ larger than the energy margin, the wave variable correction is accepted, being denoted as definitive wave correction, $v_{s 4}$. Otherwise, no modification is allowed, i.e.

$$
\begin{aligned}
& v_{s 4}(k)= \\
& \begin{cases}v_{s}^{\prime}(k) & \text { If } E M(k) \geq \sum_{i=1}^{k}\left[v_{s}^{2}(i)-v_{s 3}^{2}(i)\right] h \\
0 & \text { If } E M(k)<\sum_{i=1}^{k}\left[v_{s}^{2}(i)-v_{s 3}^{2}(i)\right] h\end{cases}
\end{aligned}
$$

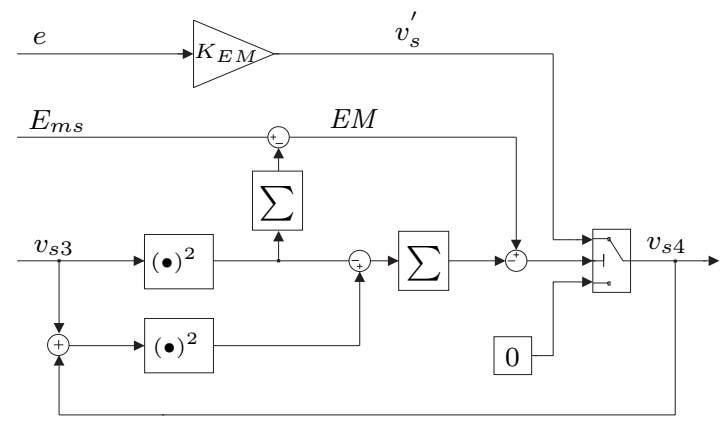

Fig. 2. Energy Margin correction

Note that, since $E_{m s}$ is nondecreasing, any delay or data loss will lead to the conservative assumption that the available energy margin is smaller than the actual one. The following pseudo-code sums up the algorithm, whose diagram is also pictured in Fig. 2:

At the Master Side:

Energy $(\mathrm{k}) \quad:=\operatorname{Energy}(\mathrm{k}-1)+\operatorname{Wave}(\mathrm{k})^{2 *} \mathrm{~h} ;$
SendPacket ( Waves, Energy, MasterPosition, k);

At the Slave Side:

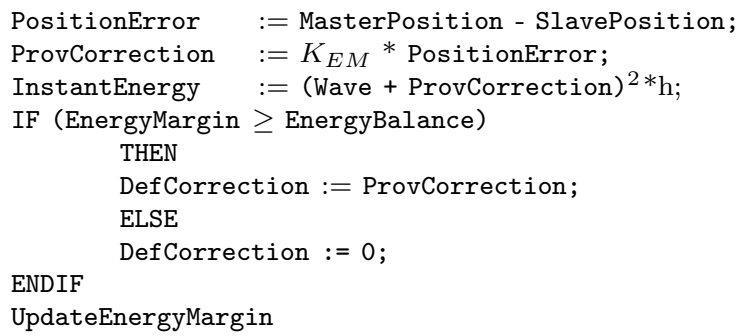




\subsection{Additional wave trimming}

In [10] it was proposed to add a corrective term to the wave command at the master side $\left(u_{m}\right)$. This correction is in principle proportional to a position error defined as $e_{2}=\left(x_{m}-x_{s d}\right)$, where $x_{m}$ is the master position, and $x_{s d}$ is the integral of $\dot{x}_{s d}$ in Fig. 1, after being sent from the master to the slave side. Thus, the correction is of the type $\delta u=K_{W T} e_{2}$, with some $K_{W T}>0$ (although in [10] a particular choice of $K_{W T}$ was suggested, it was admitted that any positive value could be chosen). In order to maintain passivity, the magnitude of the corrected wave command must be bounded by the original uncorrected version, i.e. $\left|u_{m}+K_{W T} e_{2}\right| \leq\left|u_{m}\right|$. This means that the correction is in fact "trimming" the wave variables, i.e., taking power off them. Hence, it creates an additional energy margin from which the controller described in the previous subsection can profit. In order to combine both ideas, we propose two modifications of this method:

- Perform the correction at the slave side, so that it can be implemented by the same device as the energy margin controller. Furthermore, in this way the correction is applied immediately after being calculated $\left(v_{s 2}\right.$ in Fig. 1), instead of being transmitted through the communications channel. This avoids having an additional delay $T=n_{d} h$, which is especially advantageous for large delays.

- A more general correction can be carried out if the position error is defined as $e=x_{m d}-x_{s}$ instead of $e_{2}=x_{m}-x_{s d}$, with $x_{s}$ being the position of the slave and $x_{s d}$ the integrated velocity resulting for the slave scattering transformation.

With these modifications, the control action is as follows:

$v_{s 2}= \begin{cases}0 & \text { If } v_{s 1} e>0 \\ K_{W T} e & \text { If } v_{s 1} e \leq 0 \quad \& \quad\left|K_{W T} e\right|<2\left|v_{s 1}\right| \\ -2 v_{s 1} & \text { If } v_{s 1} e \leq 0 \quad \& \quad\left|K_{W T} e\right| \geq 2\left|v_{s 1}\right|\end{cases}$

and therefore $\left|v_{s 3}(k)\right| \leq\left|v_{s 1}(k)\right|$.

\section{Controller analysis}

\subsection{Stability}

We will prove the stability of the augmented communications channel of Fig. 1, consisting of the transmission line and the controllers; i.e., from $u_{m}$ to $v_{s}$. The energy stored in a network with a delay buffer of $n_{d}$ samples, through which a wave variable $u$ is being sent is:

$E(k)=\sum_{i=k-n_{d}}^{k-1} u^{2}(i) h$
From [3,10], the network with the SIBCE (3) and WT blocks is passive (see also Assumption 1). Therefore, the line is passive from $u_{m}$ to $v_{s 3}$ (and from $u_{s}$ to $v_{m}$ ). For the transmission from master to slave the following holds:

$E(k)-E(1)=\left[\sum_{i=1}^{k-1}\left[u_{m}^{2}(i)-v_{s 3}^{2}(i)\right]-\sum_{i=1}^{k-1} d^{2}(i)\right] h$

where $\sum_{i=1}^{k-1} d^{2}(i) h$ is the dissipated energy. We need to prove that the addition of the EM block maintains passivity of the line from $u_{m}$ to $v_{s}$.

Theorem 4.1 Given the previous definitions, the line is passive between $u_{m}$ and $v_{s}$, this is:

$E(k)-E(1) \leq \sum_{i=1}^{k-1}\left[u_{m}^{2}(i)-v_{s}^{2}(i)\right] h$

Proof From (8) and (5) we have that:

$$
E(k)+E M(k)=\sum_{i=1}^{k-1}\left[u_{m}^{2}(i)-v_{s 3}^{2}(i)\right] h
$$

Let us replace with $E(k)$ from $(9)$ :

$E M(k)=-E(1)+\sum_{i=1}^{k-1} d^{2}(i) h$

Using $E(1)>0$, the condition (6), and (11):

$$
-\sum_{i=1}^{k} v_{s 3}^{2}(i) h \leq \sum_{i=1}^{k-1} d^{2}(i) h-\sum_{i=1}^{k} v_{s}^{2}(i) h
$$

If the previous inequality with $k \rightarrow(k-1)$ is replaced in (9), this yields

$$
E(k)-E(1) \leq \sum_{i=1}^{k-1} u_{m}^{2}(i) h-\sum_{i=1}^{k-1} v_{s}^{2}(i) h-d^{2}(k-1) h
$$

this is,

$$
E(k)-E(1) \leq \sum_{i=1}^{k-1}\left[u_{m}^{2}(i)-v_{s}^{2}(i)\right] h
$$

q.e.d.

\subsection{Performance}

We will analyze now the control actions in order to show how they reduce the position error. A similar analysis can be carried out for the WT and EM modules, since: 
- They are both placed at the slave side of the teleoperation setup: immediately after the communications channel and before the transformation from wave to power variables (see Fig. 1).

- The control action exerted by them is qualitatively the same, i.e. proportional to the position error, although restricted in different ways: in one case, with the limitation that the energy margin can not be exceeded; in the other, the limiting factor is the magnitude of the original wave variable.

We will refer to a scheme such as the one shown in Fig. 1 , and will use $\Delta v$ to denote the correction of the wave variable. When speaking of the WT controller, $\Delta v=v_{s 2}$, while for the EM controller $\Delta v=v_{s 4}$. For simplicity, we will examine in the first place the system in continuous time and with a constant time delay $T$. The position error is defined as the difference between the position of the master device and the setpoint arriving at the slave side. Both positions are measured at the slave side, so the master's position is delayed:

$e_{2}(t)=x_{m}(t-T)-x_{s d}(t)$

Theorem 4.2 Consider the setup in Fig. 1, with the WT and EM elements as described in section 3. Then, the effect of the corrections $v_{s 2}, v_{s 4}$ is to reduce the position error defined by (12).

Proof Let us write the derivatives of $x_{m}(t-T)$ and $x_{s d}(t)$ as functions of the wave variables, and replace $v_{m}(t-T)=u_{s}(t), v_{s}(t)=u_{m}(t-T)+\Delta v(t):$

$$
\begin{aligned}
& \dot{x}_{m}(t-T)=\frac{1}{\sqrt{2 b}} u_{m}(t-T)-\frac{1}{\sqrt{2 b}} u_{s}(t) \\
& \dot{x}_{s d}(t)=\frac{1}{\sqrt{2 b}}\left(u_{m}(t-T)+\Delta v(t)\right)-\frac{1}{\sqrt{2 b}} u_{s}(t)
\end{aligned}
$$

Hence, the position error can be written as:

$$
e_{2}(t)=x_{m}(t-T)-x_{s d}(t)=-\frac{1}{\sqrt{2 b}} \int_{0}^{t} \Delta v(\tau) d \tau
$$

Assigning a desired dynamics $\dot{e}_{2}(t)=-K e_{2}(t)$ to the error, we obtain an expression relating it to the wave variable correction:

$\Delta v(t)=K \sqrt{2 b} e_{2}(t)=K^{\prime} e_{2}(t)$

Thus, by modifying the wave variable in this way we assign to the term $x_{m}(t-T)-x_{s d}(t)$ the aforementioned dynamics. Both the energy margin controller and the wave trimmer controller use this type of correction, applying it when determined by eqs. $(6,7)$. These solutions guarantee a non increasing dynamics of the position error. If the canonical Lyapunov function $V(e)=\frac{1}{2} e_{2}^{2}$ is

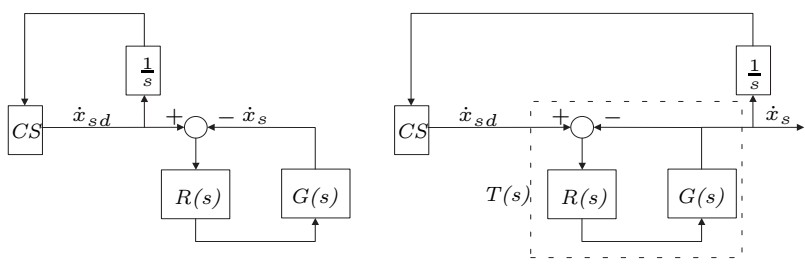

Fig. 3. Using $x_{s d}, x_{s}$

proposed, it turns out that:

$$
\dot{V}(t)=e_{2}(t) \dot{e}_{2}(t)=-e_{2}(t) \frac{1}{\sqrt{2 b}} \Delta v(t)
$$

For the WT controller, the decreasing rate for $V$ can be of three different modes, see equation (7):

$$
\dot{V}(t)=\left\{\begin{array}{l}
0 \\
-\frac{K_{W T}}{\sqrt{2 b}} e_{2}(t)^{2}=-\sqrt{\frac{2}{b}} K_{W T} V(t) \\
-e_{2}(t) \frac{1}{\sqrt{2 b}}\left(-2 v_{s 1}(t)\right)=\frac{2}{\sqrt{b}} v_{s 1}(t) \sqrt{V(t)}
\end{array}\right.
$$

Depending on the situation, the error can be left unmodified (first line), decrease strictly in an uniform way (second line), or decrease strictly as a function of $v_{s 1}$ (third line, where the sign of $v_{s 1}$ is opposite to $e_{2}(t)$ ).

For the EM controller the decrease rates (see eq. (6)) are:

$$
\dot{V}(t)=\left\{\begin{array}{l}
-e_{2}(t) \frac{1}{\sqrt{2 b}} v_{s}^{\prime}(t)=-\frac{K_{e m}}{\sqrt{2 b}} e_{2}^{2}(t) \\
0
\end{array}\right.
$$

Hence, the control actions reduce the position error as long as there is some energy available.

Remark 1. Position error The actual position error is not the $e_{2}$ given by eq. (12), but $e(t)=x_{m}(t-T)-x_{s}(t)$. The difference is shown on Fig. 3, where CS represents the Controller plus the Scattering transformation, G(s) is the transfer function of the slave device and $\mathrm{R}(\mathrm{s})$ a controller whose purpose is to make $\dot{x}_{s}$ follow $\dot{x}_{s d}$. The left part of the figure depicts the procedure that reduces $e_{2}$. The right part corresponds to the same topology as in Fig. 1, and is similar to the left one but with a transfer function between $\dot{x}_{s d}$ and $\dot{x}_{s}$. In a typical case, in which the plant is a mechanical system with mass $m_{p}$ and friction $b_{p}$, and the controller is a PI of parameters $B, K$, this transfer function takes the form $T(s)=\frac{G(s) R(s)}{1+G(s) R(s)}=\frac{B \cdot s+K}{s \cdot\left(m_{p} \cdot s+b_{p}\right)+B \cdot s+K}$. In steady state $T(0)=1$, so $\dot{x}_{s d}(\infty)=\dot{x}_{s}(\infty)$. Hence, the slave velocity tracks $\dot{x}_{s d}$, and the scheme proposed in the right part of Fig. 3 allows to decrease the position error $x_{m}-x_{s}$, as the one in the left decreases $x_{m}-x_{s d}$. 
Remark 2. Time-varying delay Theorem 4.2 can be adapted to a variable delay $T=T(t)$. From (1):

$$
\begin{aligned}
& \dot{x}_{m}(t-T)=\frac{1}{\sqrt{2 b}}\left(u_{m}(t-T)-v_{m}(t-T)\right) \\
& \dot{x}_{s d}(t) \quad=\frac{1}{\sqrt{2 b}}\left(v_{s}(t)-u_{s}(t)\right)
\end{aligned}
$$

The problem with a time-varying $T(t)$ is that $v_{m}(t-T) \neq$ $u_{s}(t)$ and $v_{s}(t) \neq u_{m}(t-T)+\Delta v$, so the derivation in Theorem 4.2 cannot be exactly applied. However, this can be solved if we consider the effect of the SIBCE and WT blocks. The SIBCE can be interpreted in continuous time [3] as a scaling by a time-varying gain: $v_{m}(t-T)=$ $u_{s}(t) \sqrt{1-\dot{T}}=: u_{s}(t) f(t)$, and similarly for $v_{s}(t)$. If we consider the delay as $T(t)=T_{0}+\delta(t)$, then in nominal conditions $(\delta(t) \equiv 0)$ we have $\dot{T}=0$ and $f(t)=1$. The WT block imposes the restriction $\left|v_{s 3}(t)\right| \leq\left|v_{s 1}(t)\right|$, and thus $v_{s 3}(t)=v_{s 1}(t) g(t)$, with $g(t)$ a known function satisfying $|g(t)| \leq 1$. Combining the effect of both blocks, eq. (14) results in:

$$
\begin{aligned}
& \dot{x}_{m}(t-T)=\frac{1}{\sqrt{2 b}}\left(u_{m}(t-T)-f(t) u_{s}(t)\right) \\
& \dot{x}_{s d}(t)=\frac{1}{\sqrt{2 b}}\left(g(t) f(t) u_{m}(t-T)+v_{s 4}-u_{s}(t)\right)
\end{aligned}
$$

Notice that, if $T=T(t)$, the derivative of $e_{2}$ is:

$\dot{e}_{2}(t)=\dot{x}_{m}(t-T)(1-\dot{T})-\dot{x}_{s d}(t)=\dot{x}_{m}(t-T) f^{2}(t)-\dot{x}_{s d}(t)$

Using (15) gives:

$\dot{e}_{2}(t)=\frac{1}{\sqrt{2 b}}(\underbrace{f(f-g) u_{m}(t-T)+\left(1-f^{3}\right) u_{s}(t)}_{=: h(t)}-f g \Delta v)$

Notice that in nominal conditions $\left(T(t) \equiv T_{0}\right)$ we have $f(t) \equiv 1$, and if the WT block is not used $g(t) \equiv 1$, so that $h(t) \equiv 0$ and $\dot{e}_{2}(t)=\frac{-1}{\sqrt{2 b}} \Delta v$, as previously. In any case, for the general situation, since $h(t)$ is a known function, we can postulate the wave correction

$$
\Delta v(t)=\frac{h(t)+\sqrt{2 b} K e_{2}(t)}{f(t) g(t)}
$$

which, applied according to (6), gives rise to the desired target dynamics $\dot{e}_{2}=-K e_{2}$.

Remark 3. Discrete time Until now the problem has been formulated in continuous time. In discrete time the proof is similar, starting from the definition of the error of eq. (4). There, $x_{m d}(k)$ represents the position of the master device after being transmitted through the communications channel, hence delayed by a certain number of sampling intervals, corresponding to the continuous time delay $T=n_{d} h$. Similarly, and writing the derivative of a variable $x(k)$ as $d x(k)=\frac{x(k+1)-x(k)}{T}$, we have:

$$
\begin{aligned}
& d x_{m d}(k)=\frac{1}{\sqrt{2 b}} u_{m d}(k)-\frac{1}{\sqrt{2 b}} u_{s}(k) \\
& d x_{s}(k)=-\frac{1}{\sqrt{2 b}} u_{s}(k)+\frac{1}{\sqrt{2 b}}\left(u_{m d}(k)+\Delta v_{m}(t)\right)
\end{aligned}
$$

Obtaining for the wave correction an expression similar to (13), $\Delta v(k)=K^{\prime} e_{2}(k)$. Therefore the proposed controllers can be implemented in discrete time.

Remark 4. Asymptotic convergence of the error The proposed error correction schemes impose a stable dynamics $\dot{e}_{2}=-K e_{2}$ with $K=K_{W T}$ or $K=K_{E M}$, so that $\dot{V}=e_{2} \dot{e}_{2}=-K e_{2}^{2}$. The problem is that, in order to maintain passivity, it is not always possible to apply the desired wave corrections, but only when the first inequality in (6) or the second one in (7) hold. Fortunately, when these conditions do not hold, the error is at least not increasing: $\dot{V} \leq 0$. Furthermore, it can be expected that those conditions have to be feasible part of the time. In particular, the energy margin of the EM block is being continuosly charged, so sooner or later the wave correction in (6) will be enabled. It is very difficult to reach without assumptions a formal, analytical proof of the convergence $e_{2} \rightarrow 0$ within the global system in Fig. 1, for it is a complex, distributed (time-delay), hybrid (continuous-discrete), and nonlinear (WT and EM blocks) system. However, there is a direct justification under a very mild, reasonable assumption:

Assumption 2 Suppose there exists a time window with a (large enough) $W>0$ and a (small enough) number $0<p \leq 1$, such that for every $t \geq 0$ in each interval $[t, t+\bar{W}]$, the wave correction in $(\overline{6})$ is enabled at least $(100 p) \%$ of the time, that is, at least during $p \cdot W$ seconds.

Under this assumption, $\dot{V}(t) \leq-K e_{2}^{2}=-2 K V(t)$ holds for at least $p \cdot W$ seconds and $\dot{V} \leq 0$ for at most $(1-$ p) $W$ seconds. Consider without loss of generality that the "enabled" time is the first part of the interval, $[t, t+$ $p \cdot W]$; the worst case behaviour for $[t+p \cdot W, t+W]$ is $\dot{V}=0$, so:

$$
V(t+W) \leq V(t+p W) \leq V(t) e^{-2 K(p W)}
$$

In conclusion, the error does not always decrease strictly at every $t$, but considering intervals of length $W$ there is an asymptotic convergence to zero with a coefficient $K \cdot p$, that is, the assigned coefficient $K$ times $p>0$, where $100 \mathrm{p} \%$ is the percentage of time when it is expected that the energy margin provides correction capabilities.

\section{Experiments and Comments}

The two controllers can be integrated in a teleoperation setup as in Fig. 1. After being transmitted through 
the network, the arriving wave variable $\left(v_{s 1}\right)$ is modified using the Wave Trimmer module (WT). Then, the energy margin-generated at the network and by the WT-is used by the EM module to add another corrective term $\left(v_{s 4}\right)$. The resulting wave variable $\left(v_{s}\right)$ is transformed into a velocity command $\dot{x}_{s d}$, and a PI control action is applied: $F_{s}=\left(B_{c}+\frac{K_{c}}{s}\right)\left(\dot{x}_{s d}-\dot{x}_{s}\right)$. In order to test the performance of this scheme, several simulations were carried out in a Matlab/Simulink environment. Communications via UDP were simulated with the Handshake ProSense Virtual Touch Toolbox (Network Option). An average delay of 3 seconds, with a standard deviation of 0.5 and a $20 \%$ packet loss was forced for each channel (master to slave and slave to master). SensAble Technologies' PHANTOM Omni haptic device was used as the master, and a second order plant with transfer function $\frac{1}{M_{p} s^{2}+B_{p} s}$ was used as the slave. A rigid wall at $x_{s}=0.3$ was simulated at the remote environment. The simulation parameters were $b=B_{p}=B_{c}=10[\mathrm{~kg} / \mathrm{s}]$, $M_{p}=5[\mathrm{~kg}], K_{c}=10\left[\mathrm{~kg} / \mathrm{s}^{2}\right], K_{E M}=5\left[\mathrm{~kg} / \mathrm{s}^{2}\right]$.

The goal of the teleoperation setup is to make the slave device track the master device's trajectory. Additionally, we want it to correct any initial position mismatch between master and slave, so that in steady state both positions are identical. In a standard teleoperation setup without additional position error correction, the initial position mismatch between master and slave cannot be eliminated, see Fig. 4. By adding a wave trimmer module at the master side as in [10] the slave tries to reduce this error, but is not able to do it after the master stops (Fig. 5). In Fig. 6 it is shown how the method proposed in [16] improves position tracking, but it's not able to eliminate the initial mismatch. Finally, with the combined application of the EM and WT control actions, the steady state position error is made zero (Fig. 7). See [6] for results of applying the proposed correction to a teleoperated gantry crane.

Regarding force feedback, Fig. 8 shows that the proposed method also maintains transparency. It's divided in 4 subplots: those on the left side (8.A, 8.B) correspond to a bilateral teleoperation setup that includes the arrangement in [3] for maintaining passivity in UDPbased communications. Those on the right side (8.C, 8.D) include additionally the position correction method presented here. In these simulations, the operator applies a triangular-shaped force command, while there's a square-shaped environmental contact force at the slave side. These forces are shown on the upper subplots (8.A, 8.C). The force fed back to the master side, $F_{m}$, is shown in the lower subplots (8.B, 8.D); and it can be seen that in both cases it matches the composition of command and contact forces, as expected. The network conditions in this case were those of a UDP-based communication with normal distributed latency, with an average delay of 1 second from master to slave and from slave to master, an standard deviation of 0.001 seconds, and a $10 \%$ of packet losses.

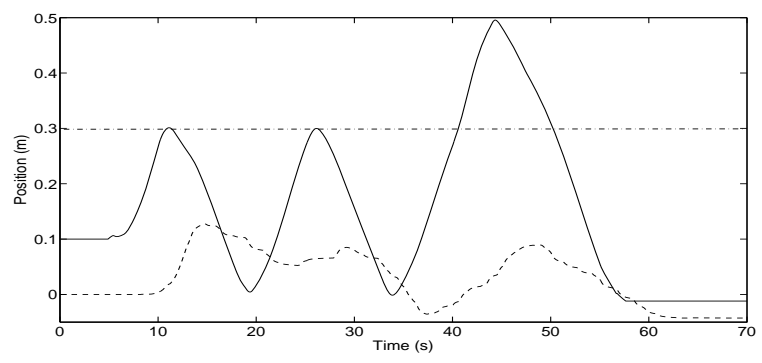

Fig. 4. Teleoperation without position correction (solid: master, dashed: slave, horizontal line: wall)

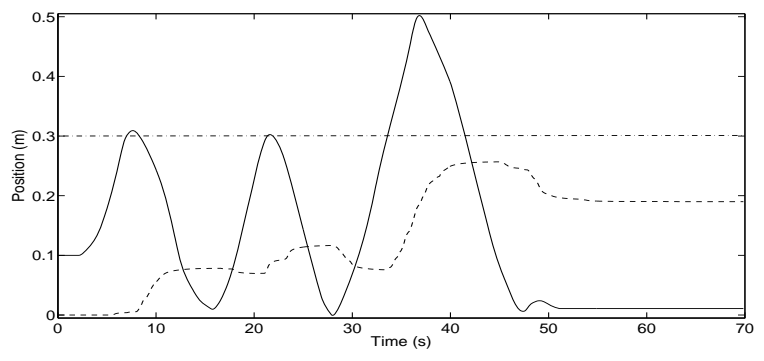

Fig. 5. Wave trimmer by Niemeyer \& Slotine (solid: master, dashed: slave, horizontal line: wall)

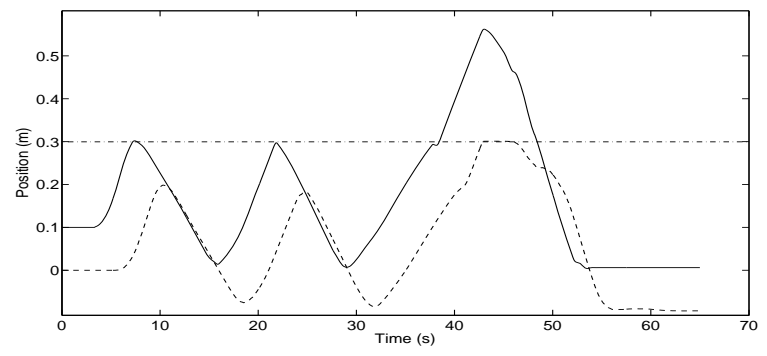

Fig. 6. Method by Yokohkoji et al.

(solid: master, dashed: slave, horizontal line: wall)

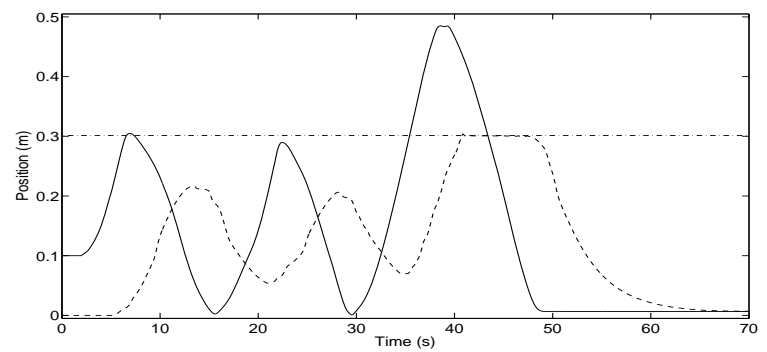

Fig. 7. EM + WT

(solid: master, dashed: slave, horizontal line: wall)

We have mentioned the method in [16] and compared it experimentally with the one presented here. We shall briefly comment their similarities and differences, since both of them are partially inspired by [10]. One difference is that [16] is TCP/IP-based: it assumes that the data packets arrive in the original order. Our method, however, is UDP-based, and therefore makes no assump- 


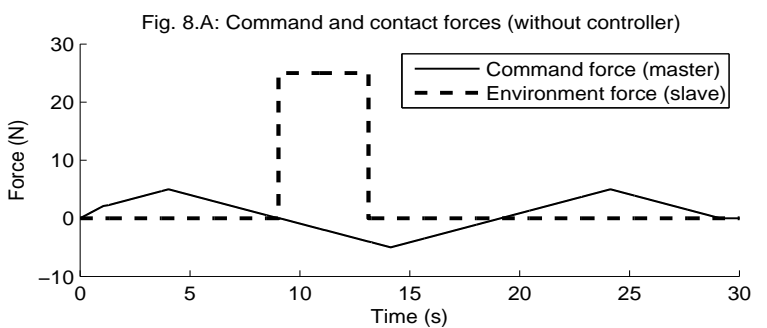

Fig. 8.B: Feedback force (without controller)

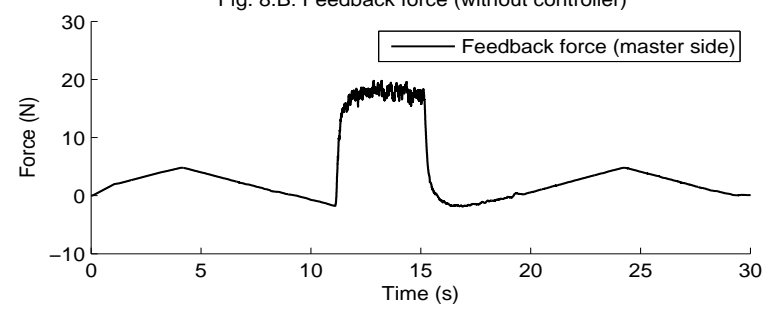

Fig. 8.C: Command and contact forces (with TW + EM)
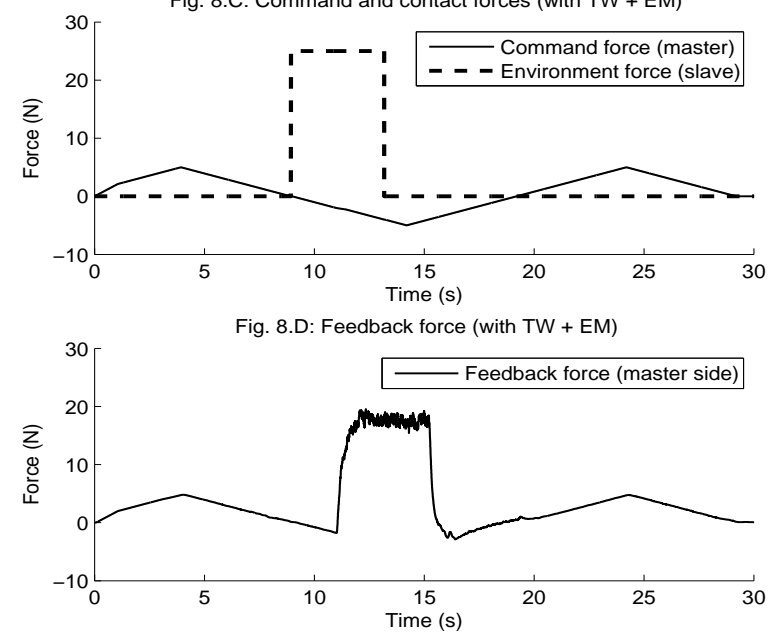

Fig. 8. Contact and transmitted forces. Left: without the proposed controller; right: with the proposed controller.

tion about that point. Another difference is that in [16] passivity is not strictly preserved, since a "last packet" strategy is adopted instead of a "null packet" one (see section 2). This, on the one hand, makes the method less conservative, although on the other hand additional arrangements for guaranteeing stability are required. Finally, in [16] no position measurements are used; hence, the goal of the algorithm is to restore the original wave variable, but it cannot correct position errors caused by an initial position mismatch, as shown on Fig. 6 .

Another method that has some similarities with the present one is [15]. Both implement some "energy tank", charged by dissipated energy, that is reused to eliminate position errors. Our proposal stores the energy dissipated in the Internet line from master to slave (including the wave trimming block, WT). The proposal by [15] stores the energy dissipated in the line from master to slave (no WT block) and from slave to master, as well as the energy dissipated in the slave controller. Regarding this issue, they are complementary: [15] could be adapted to include energy from a WT block, and our solution could be adapted to include energy from the slave to master line and from the slave controller. One difference is that, while our proposal is based on wave variables, [15] is applied to power variables (flows and efforts), and hence it requires some connection matrix for the reuse of energy for position error correction. Its tuning, as well as the relation with position errors, is not formally clarified in detail. On the other hand, our proposal implements corrections directly to the wave variables as in (7), it is based on (see Theorem 4.2) a explicit Lyapunov function (not presented in [15]) and it can be easily adapted (Remark 2) to time-varying delays. Regarding energy storage, our solution simply accumulates the wave packet balance, whereas [15] requires an extra flow parameter to adjust the speed at which the tank is charged. Putting aside the differences, both techniques are formally correct and can be augmented to include energy dissipated by other sources in the global system (plant, controllers, line,...). It is not possible to present here a comparison based on numerical simulations since there are a number of parameters in $[15]\left(f_{L}, \pm \epsilon, A, \ldots\right)$ that require careful expert tuning, which is beyond the scope of this work.

\section{Conclusions}

We have presented a controller that improves position tracking in UDP-based bilateral teleoperation, by exploiting the energy margin resulting from passivation of the communications channel. Since the available energy margin increases with the unreliability of the network, the method is especially suitable for communications with large, unpredictably varying delays and loss of data. The passivity framework guarantees robust stability of the teleoperation setup under any condition. Theoretical and experimental results have been provided in order to support the choice of the two modules of the proposed controller.

\section{Acknowledgements}

Research supported by the Spanish Ministry of Education and Science under grant DPI2007-66455-C02-02.

\section{References}

[1] R.J. Anderson and M.W. Spong. Bilateral control of teleoperators with time delay. IEEE Trans. Automat. Contr., 5:494-501, 1989.

[2] A. Aziminejad, M. Tavakoli, R.V. Patel, and M. Moallem. Stability and performance in delayed bilateral teleoperation: Theory and experiments. Contr. Eng. Pract., 16:1329-1343, 2008. 
[3] N. Chopra, P. Berestesky, and M.W. Spong. Bilateral teleoperation over unreliable communication networks. IEEE Trans. Contr. Syst. Tech., 16:304-313, 2008.

[4] N. Chopra, M. W. Spong, R. Ortega, and N. E. Barabanov. On tracking performance in bilateral teleoperation. IEEE Trans. Robotics, 22(4):861-866, 2006.

[5] N. Chopra, M.W. Spong, and R. Lozano. Synchronization of bilateral teleoperators with time delay. Automatica, 44:21422148, 2008.

[6] A. Fernández Villaverde, A. Barreiro, and C. Raimúndez. Haptic interface for crane teleoperation. In Proc. of the CONTROLO'08, pages 46-51, 2008.

[7] P.F. Hokayem and M.W. Spong. Bilateral teleoperation: An historical survey. Automatica, 42:2035-2057, 2006.

[8] S. Munir and W. J. Book. Internet-based teleoperation using wave variables with prediction. IEEE/ASME Trans. on Mechatronics, 7(2):124-133, 2002.

[9] G. Niemeyer and J-J.E. Slotine. Stable adaptive teleoperation. IEEE J. Ocean. Eng., 16(1):152-162, 1991.

[10] G. Niemeyer and J.-J.E. Slotine. Designing force reflecting teleoperators with large time delays to appear as virtual tools. In Proc. of the ICRA, volume 3, pages 2212-2218, 1997.

[11] G. Niemeyer and J.-J.E. Slotine. Using wave variables for system analysis and robot control. In Proc. of the ICRA, volume 2, pages 1619-1625, 1997.

[12] E. Nuño, L. Basáñez, and R. Ortega. Passive bilateral teleoperation framework for assisted robotic tasks. In Proc. of the ICRA, pages 1645-1650, 2007.

[13] E. Nuño, R. Ortega, and Basánez. An adaptive controller for nonlinear teleoperators. Automatica, 46:155-159, 2010.

[14] C. Secchi, S. Stramigioli, and C. Fantuzzi. Position drift compensation in port-hamiltonian based telemanipulation. In IEEE/RSJ Int. Conf. on Intelligent Robots and Systems, pages 4211-4216, 2006.

[15] C. Secchi, S. Stramigioli, and C. Fantuzzi. Compensation of position errors in passivity based teleoperation over packet switched communication networks. In Proc. of the 17th IFAC World Congress, pages 15648-15653, 2008.

[16] Y. Yokokohji, T. Tsujioka, and T. Yoshikawa. Bilateral control with time-varying delay including communication blackout. In Proc. of the 10th Symp. on Haptic Interfaces for Virtual Environ. and Teleop. Systems, pages 285-292, 2002. 